\title{
Caracterização hidrodinâmica de solos: Aplicação do método Beerkan ${ }^{1}$
}

\author{
Eduardo S. de Souza ${ }^{2}$, Antonio C. D. Antonino ${ }^{3}$, Rafael Angulo-Jaramillo ${ }^{4}$ \& André Maciel Netto ${ }^{3}$
}

\begin{abstract}
RESUMO
O conhecimento das propriedades hidráulicas do solo, tais como as curvas de retenção da água no solo $\theta$ (h) e da condutividade hidráulica $K(\theta)$, é indispensável para a modelagem do transporte de água e de poluentes em sistemas agrícolas. Várias técnicas experimentais têm sido propostas para determinar essas características do solo diretamente no campo, porém a complexidade, os custos elevados e o tempo de execução dessas medidas, são fatores que limitam bastante a obtenção dessas informações em grande escala. Apresenta-se, no presente trabalho, uma metodologia conhecida como "Beerkan", com a qual se busca minimizar esses fatores utilizando-se dados experimentais de infiltração, da distribuição granulométrica e da massa específica do solo, para então se estimar os parâmetros das curvas de $\theta(h)$ e $K(\theta)$. O método foi aplicado em dois solos com diferentes classes texturais (três de um Latossolo Amarelo e três de um Neossolo Flúvico). Determinaram-se, com vistas à caracterização hidrodinâmica, os parâmetros de forma das curvas de $\theta$ (h) de van Genuchten e de $K(\theta)$ de Brooks e Corey, dependentes majoritariamente da textura do solo e obtidos a partir da curva de distribuição dos tamanhos das partículas, além dos parâmetros de normalização dependentes, essencialmente, da estrutura do solo e obtidos a partir dos ensaios de infiltração utilizando-se o infiltrômetro de anel simples. O método Beerkan provou ser robusto e adaptado à caracterização hidrodinâmica dos dois solos.
\end{abstract}

Palavras-chave: parâmetros hidrodinâmicos, solo não saturado, infiltração

\section{Hydrodynamic characterization of soils: Application of the Beerkan method}

\begin{abstract}
Knowledge of the soil hydraulic properties, such as the water retention $\theta(\mathrm{h})$ and hydraulic conductivity $K(\theta)$ curves, is indispensable for water and pollutant transport modeling of agricultural systems. Several experimental techniques have been proposed to determine these characteristic curves of the soil in the field. However, the complexity, the high costs and the time of execution of measurements are limiting factors for obtaining these characteristic curves in large areas. In this work, a simple methodology is shown, known as "Beerkan", which secks to overcome these difficulties. It uses experimental data of infiltration, bulk density and the particle-size distribution for estimating the $\theta(h)$ and $K(\theta)$ curves. This method was applied to two soils with different textural classification (three of an Oxisoil and three of an Alluvial Soil). For the hydrodynamic characterization, the shape and the normalization parameters of the $\theta(h)$ and of $K(\theta)$ curves were determined. The shape parameters depend upon the soil texture and they were obtained from the cumulative particlesize distribution curve; the normalization parameters depend on soil structure and they were obtained from the infiltration experiments using the simple ring infiltrometer. The Beerkan method proved to be robust and adapted to modeling the three-dimensional infiltration in field, as well as allowing the hydrodynamic characterization of the two soils.
\end{abstract}

Key words: hydrodynamic parameters, unsaturated soil, infiltration

\footnotetext{
1 Parte da Tese de Doutorado do primeiro autor, apresentada ao PROTEN/DEN, UFPE

${ }^{2}$ CCA/UFPB, Cidade Universitária, CEP 58397-000, Areia, PB. Fone: (83) 3362-2300, Ramal: 296. E-mail: eduardossouza@hotmail.com

3 DEN/UFPE, Av. Prof. Luís Freire 1000, Cidade Universitária, CEP 50740-540, Recife. Fone: (81) 2126-8252. E-mail: acda@ufpe.br; amnetto@ufpe.br

${ }^{4}$ Pesquisador do Laboratoire des Sciences de l'Environnment, ENTPE, Rue Maurice Audin, 69518 Vaulx en Vélin, França. E-mail: angulo@hmg.inpg.fr
} 


\section{INTRODUÇÃO}

A descrição ou predição dos processos da dinâmica da água e de sais no solo em condições de campo (em processos de irrigação, drenagem, conservação de água, recarga e contaminação do lençol freático, e infiltração e escoamento superficial) necessita do conhecimento das características hidrodinâmicas do solo, ou seja: i) da relação entre o potencial matricial (h) e a umidade volumétrica do solo $\theta(\mathrm{h})$, conhecida como curva característica da umidade do solo ou curva de retenção da água no solo, e ii) da relação entre a condutividade hidráulica e a umidade volumétrica $K(\theta)$, conhecida como curva de condutividade hidráulica (Hillel, 1998).

Normalmente, para se fazer inferências sobre o valor verdadeiro de uma propriedade do solo no campo, é necessário coletar um grande número de informações. No caso da caracterização hidrodinâmica dos solos isto implica em ensaios de campo e de laboratório bastante dispendiosos, oneram e demandam um longo tempo de execução dos experimentos. O custo efetivo dessas determinações levou alguns pesquisadores a utilizarem métodos indiretos que se baseiam em dados do solo prontamente disponíveis, usuais e de baixo custo (Wagner et al., 2001; Minasny et al., 1999; Minasny \& Mac Bratney, 2002); além disso, teorias e modelos matemáticos têm sido desenvolvidos para descrever com propriedade o movimento da água no solo (Haverkamp et al., 1998).

Um método semifísico, denominado "Beerkan" (Haverkamp et al., 1998; Braud et al., 2005; Lassabatère et al., 2006), propõe a estimativa dos parâmetros das curvas $\theta(h)$ e $K(\theta)$ considerando a textura e a estrutura do solo; neste método, $\theta(h)$ e $K(\theta)$ podem ser descritas, analiticamente, por cinco parâmetros: dois de forma, m ou $n$ e $\eta$, relacionado principalmente com a textura, e três de normalização $\theta_{\mathrm{s}}, \mathrm{K}_{\mathrm{s}}$ e $\mathrm{h}_{\mathrm{g}}$, dependentes da estrutura do solo. Os parâmetros de forma são obtidos a partir da curva de distribuição dos tamanhos das partículas $F(D)$ e da porosidade, enquanto os parâmetros de normalização são determinados a partir de experimentos de infiltração.

Os parâmetros de normalização apresentam importantes variações espaço-temporal ligados à estrutura do solo (Haverkamp et al., 1998); desta forma, eles não devem ser calculados a partir de simples caracterizações texturais, como ocorre na maioria das funções de pedotransferência. Uma alternativa para analisar as variações estruturais é através de medidas do fluxo de infiltração. O método Beerkan propõe a obtenção da curva de infiltração acumulada em função do tempo, com um infiltrômetro a disco ou com um infiltrômetro de anel simples. A referida metodologia permite determinar esses parâmetros hidrodinâmicos do solo, independentemente da escala de estudo (de Condappa et al., 2002; Braud et al., 2005; Lassabatère et al., 2006).

Objetivou-se, neste trabalho, determinar e analisar os parâmetros de forma e de normalização das curvas $\theta(h)$ e $K(\theta)$, por intermédio do método "Beerkan", em dois solos, apresentando três distintas classes texturais, do Estado da Paraíba.

\section{MATERIAL E MÉTODOS}

O estudo foi realizado em duas parcelas localizadas nos municípios de Areia (6 $58^{\prime} 12^{\prime \prime} \mathrm{S}$ e $35^{\circ} 42^{\prime} 15^{\prime}$ ' O) e São João do Cariri ( $7^{\circ} 23^{\prime} 27^{\prime}$ ' S e 36 31' 58” O), Estado da Paraíba. Os solos das parcelas são classificados, respectivamente, como Latossolo Amarelo (LA) e um Neossolo Flúvico (NF) (EMBRAPA, 1999). Três pontos com diferentes classes texturais foram escolhidos nos dois solos, onde também se realizaram ensaios com um infiltrômetro de anel simples de 15 cm de diâmetro e coletas de amostras de solo deformadas para determinação da curva granulométrica. Os ensaios de infiltração consistem em se anotar o tempo que volumes constantes de água (70-250 mL), adicionados continuamente no anel, levam para ser infiltrados; esse teste fornece a infiltração tridimensional axissimétrica como uma função do tempo $I_{3}(t)$. Durante cada teste se coletaram, ainda, amostras indeformadas para determinação da massa específica do solo e das umidades inicial $\theta_{0}$ e final $\theta_{\mathrm{s}}$. A análise granulométrica foi realizada utilizando-se o método da ABNT (1984), o qual permite determinar os diâmetros das partículas mais finas (argila e silte) por sedimentação e as mais grosseiras (areia) por peneiramento.

As classes texturais estudadas foram: franco-argiloso (FArg (LA)), franco-argilo-arenoso (FArgAre (LA)) e franco-arenoso (FAre (LA)) para o Latossolo Amarelo (LA); e franco-arenoso (FAre (NF)), areia-franca (ArFranca (NF)) e areia (Areia (NF)), para o Neossolo Flúvico (NF) (Figura 1).

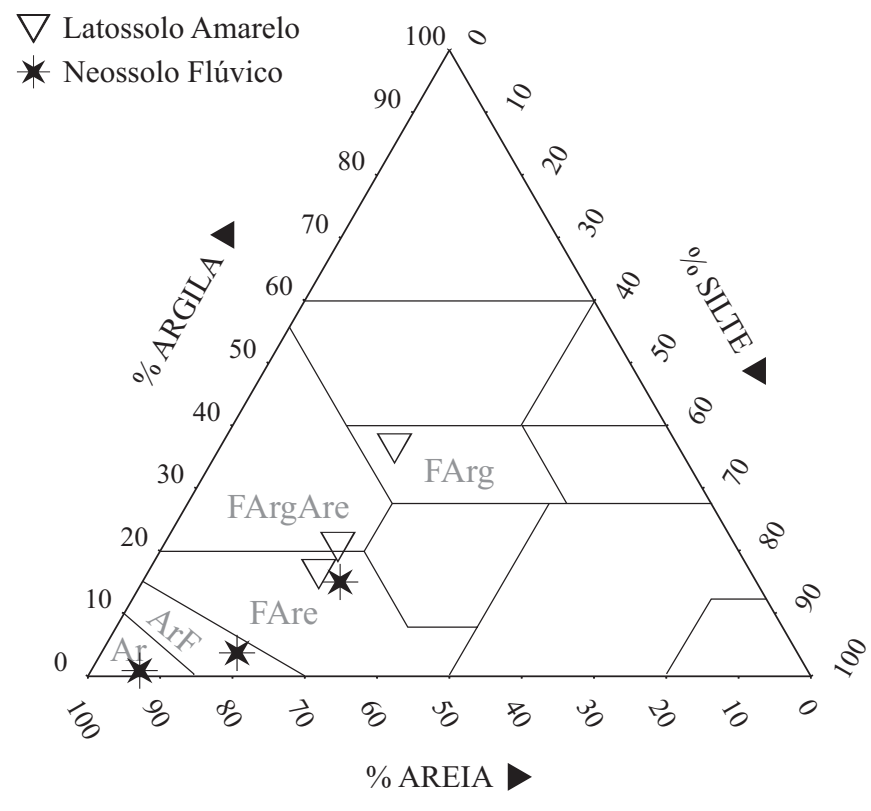

Figura 1. Diagrama das classes texturais dos solos LA e NF

\section{Metodologia Beerkan}

Neste estudo, $\theta(h)$ e $K(\theta)$ são descritas, respectivamente, pelos modelos de van Genuchten (1980) e de Brooks \& Corey (1964):

$\left(\frac{\theta-\theta_{\mathrm{r}}}{\theta_{\mathrm{s}}-\theta_{\mathrm{r}}}\right)=\left[1+\left(\frac{\mathrm{h}}{\mathrm{h}_{\mathrm{g}}}\right)^{\mathrm{n}}\right]^{-\mathrm{m}}$ com $\mathrm{m}=1-\frac{2}{\mathrm{n}}$ (Burdine, 1953) 
e

$$
\mathrm{K}(\theta)=\mathrm{K}_{\mathrm{s}} \cdot\left(\frac{\theta-\theta_{\mathrm{r}}}{\theta_{\mathrm{s}}-\theta_{\mathrm{r}}}\right)^{\eta}
$$

sendo $\theta$ a umidade volumétrica $\left[\mathrm{L}^{3} \mathrm{~L}^{-3}\right] ; \theta_{\mathrm{r}}$ e $\theta_{\mathrm{s}}$ as umidades volumétricas residual e saturada $\left[\mathrm{L}^{3} \mathrm{~L}^{-3}\right]$, respectivamente; $h$ o potencial matricial [L]; $h_{\mathrm{g}}$ [L] um valor de escala de $h ; m$ e n são parâmetros de forma; $K_{\mathrm{s}}$ a condutividade hidráulica saturada do solo [ $\mathrm{L} \mathrm{T}^{-1}$ ] e $\eta$ o parâmetro de forma para a curva de condutividade hidráulica.

Essas funções contêm cinco parâmetros desconhecidos: dois parâmetros de forma (m ou n e $\eta$ ) e três parâmetros de normalização $\left(\theta_{s}, K_{s}\right.$ e $\left.h_{g}\right)$. Os parâmetros de forma dependem principalmente da textura (Haverkamp et al., 1998), enquanto os parâmetros de normalização resultam da estrutura dos solos. Obtiveram-se os parâmetros de forma e normalização usando-se o programa BEST (Beerkan Estimation of Soil Transfer Parameters through Infiltration Experiments) proposto por Lassabatére et al. (2006).

\section{BEST - Algoritmo e análise dos dados (Lassabatère et al., 2006)}

\section{Determinação dos parâmetros de forma (m, n e $\eta$ )}

Assumindo a similaridade de forma entre a curva de distribuição do tamanho das partículas F(D) e da curva de retenção da água no solo $\theta(\mathrm{h})$, Haverkamp \& Parlange (1986) apresentaram a seguinte equação para expressar F(D):

$$
F(D)=\left[1+\left(\frac{D_{g}}{D}\right)^{N}\right]^{-M} \operatorname{com} M=1-\frac{2}{N}
$$

sendo D o diâmetro da partícula [L], Dg o parâmetro de escala do tamanho das partículas [L] e M e N os parâmetros de forma da curva de distribuição do tamanho das partículas.

Os parâmetros de forma da curva de retenção m e n são obtidos a partir do índice de forma do meio $\mathrm{p}_{\mathrm{m}}$ pelas seguintes relações:

$$
\begin{aligned}
& \mathrm{m}=\frac{1}{\mathrm{p}_{\mathrm{m}}}\left(\sqrt{1+\mathrm{p}_{\mathrm{m}}{ }^{2}}-1\right) \\
& \mathrm{n}=\frac{2}{1-\mathrm{m}}
\end{aligned}
$$

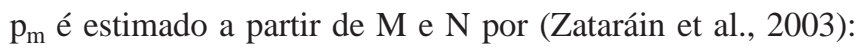

$$
\mathrm{p}_{\mathrm{m}}=\frac{\mathrm{MN}}{1+\mathrm{M}}(1+\kappa)^{-1}
$$

sendo $\kappa$ um coeficiente definido por Fuentes et al. (1998):

$$
\kappa=\frac{2 \mathrm{~s}-1}{2 \mathrm{~s}(1-\mathrm{s})}
$$

e s a dimensão fractal relativa. A dependência de $s\left(s=D_{f} / E\right.$, na qual $D_{\mathrm{f}}$ é a dimensão fractal do solo e $\mathrm{E}=3$ a dimensão de Euclides) com respeito à porosidade total do solo $(\phi)$ é definida por (Fuentes, 1992):

$$
(1-\phi)^{\mathrm{s}}+\phi^{2 \mathrm{~s}}=1 \quad \text { com } \quad 1 / 2<\mathrm{s}<1
$$

No caso da equação da curva de condutividade hidráulica de Brooks \& Corey (1964), o seu parâmetro de forma, ๆ, pode ser expresso como função do produto dos parâmetros de forma da curva de retenção e do fator de tortuosidade (p):

$$
\eta=\frac{2}{m n}+2+p
$$

sendo $\mathrm{p}=1$ para o modelo de Burdine (1953).

\section{Determinação dos parâmetros de normalização $\left(\boldsymbol{\theta}_{\mathrm{s}}, \mathrm{K}_{\mathrm{s}} \mathbf{e} \mathbf{h}_{\mathrm{g}}\right)$}

O primeiro parâmetro de normalização, $\theta_{\mathrm{s}}$, foi estimado a partir da umidade gravimétrica saturada e da massa específica do solo; os outros parâmetros de normalização são obtidos a partir da modelagem dos experimentos de infiltração. Para uma fonte de água circular com dado potencial de pressão da água sobre uma superfície de solo uniforme e com um conteúdo de água inicial uniforme $\left(\theta_{0}\right)$, a infiltração acumulada tridimensional I(t) e a taxa de infiltração $\mathrm{q}(\mathrm{t})$ podem ser aproximadas pelas equações para o regime de fluxo transitório (Eqs. 10 e 11) e estacionário (Eqs. 12 e 13) (Haverkamp et al., 1994):

$$
\begin{aligned}
& I(t)=S \sqrt{t}+\left[a S^{2}+b K_{s}\right] t \\
& q(t)=\frac{S}{2 \sqrt{t}}+\left[a S^{2}+b K_{s}\right] \\
& I_{+\infty}(t)=\left[a S^{2}+K_{s}\right] \cdot t+c \frac{S^{2}}{K_{s}} \\
& q_{+\infty}(t)=q_{+\infty}=a S^{2}+K_{s}
\end{aligned}
$$

com,

$$
\begin{aligned}
& \mathrm{a}=\frac{\gamma}{\mathrm{r} \Delta \theta} \\
& \mathrm{b}=\left(\frac{\theta}{\theta_{\mathrm{s}}}\right)^{\eta}+\frac{2-\beta}{3} \cdot\left(1-\left(\frac{\theta}{\theta_{\mathrm{s}}}\right)^{\eta}\right) \\
& \mathrm{c}=\frac{1}{2\left[1-\left(\frac{\theta}{\theta_{\mathrm{s}}}\right)^{\eta}\right] \cdot(1-\beta)} \cdot \ln \left(\frac{1}{\beta}\right)
\end{aligned}
$$

sendo $\mathrm{S}$ a sorvidade [ $\mathrm{L} \mathrm{T}^{-1 / 2}$, r o raio do cilindro [L]; $\gamma$ igual a 0,75 e $\beta$ igual a 0,6 .

Para determinação de $\mathrm{K}_{\mathrm{s}}$ e $\mathrm{S}$, o BEST utiliza equações equivalentes às Eqs. 10 e 11, obtidas pela substituição de $K_{s}$ em função da sorvidade $S$ e da taxa de infiltração no regime estacionário $\mathrm{q}_{+\infty}$, (Eq.13) nas Eqs. 10 e 11:

$$
\begin{aligned}
& \mathrm{I}(\mathrm{t})=\mathrm{S} \sqrt{\mathrm{t}}+\left[\mathrm{a}(1-\mathrm{b}) \cdot \mathrm{S}^{2}+\mathrm{bq}_{+\infty}\right] \mathrm{t} \\
& \mathrm{q}(\mathrm{t})=\frac{\mathrm{S}}{2 \sqrt{\mathrm{t}}}+\left[\mathrm{a}(1-\mathrm{b}) \cdot \mathrm{S}^{2}+\mathrm{bq}_{+\infty}\right]
\end{aligned}
$$

O ajuste da Eq. 17 aos dados experimentais da infiltração acumulada $\left(\mathrm{I}_{\exp }(\mathrm{t})\right)$ é obtido pela minimização da clássica função objeto, dada por: 


$$
f_{I}\left(S, K_{s}, k\right)=\sum_{i=1}^{k}\left[I_{\text {exp }}\left(t_{i}\right)-I\left(t_{i}\right)\right]^{2}
$$

na qual k é o número considerado de pontos no regime transitório. $\mathrm{O}$ algoritmo usado na minimização de $\mathrm{f}_{\mathrm{I}}\left(\mathrm{S}, \mathrm{K}_{\mathrm{s}}, \mathrm{k}\right)$ é o de Marquardt (1963) e o desempenho dos ajustes é analisado pelos valores correspondentes ao erro quadrático médio (EQM):

$$
E Q M=\sqrt{\frac{\sum_{i=1}^{k}\left[I_{\exp }\left(t_{i}\right)-I\left(t_{i}\right)\right]^{2}}{\sum_{i=1}^{k}\left(I_{\exp }\left(t_{i}\right)\right)^{2}}}
$$

Inicialmente, o BEST estima um valor máximo para a sorvidade, $\mathrm{S}_{\text {MAX }}$, a partir do ajuste dos dados experimentais com a Eq. 17, caso em que se assume um fluxo de água dependente apenas da capilaridade, considerando-se b igual a zero.

Como as Eqs. 10, 11, 17 e 18 são válidas apenas para o regime transitório, é possível que os ajustes não sejam feitos para todos os valores de k; a sorvidade é, então, estimada para valores de $\mathrm{k}$ de no mínimo cinco pontos para um máximo de $\mathrm{N}_{\text {tot }}$. $\mathrm{S}_{\mathrm{MAX}}$ é considerada o valor máximo de toda a sequência de pontos. Para se obter valores positivos de $\mathrm{K}_{\mathrm{s}}$, levam-se em consideração as seguintes condições: $S_{\text {MAX }}{ }^{2}$ deve ser menor que a taxa de infiltração no regime estacionário $\left(\mathrm{q}_{+\infty}\right)$ dividido pelo coeficiente a (Eq. 14); assim, a sorvidade máxima $\mathrm{S}_{\text {MAX }}$ é definida por:

$$
\mathrm{S}_{\mathrm{MAX}}=\underset{\mathrm{N}_{\mathrm{oss}}=1}{\operatorname{MAX}} \underset{\mathrm{N}_{\mathrm{wos}}-1}{ }\left[\operatorname{MIN}\left(\mathrm{S}_{\mathrm{Nobs}}(\mathrm{b}=0), \sqrt{\frac{\mathrm{q}_{+\infty}}{\mathrm{a}}}\right)\right]
$$

Para obtenção de $K_{s}$, considera-se o valor verdadeiro de b (Eq. 15). O ajuste é executado minimizando a função objeto definida pela Eq. 19. Como os ajustes pela Eq. 17 nem sempre são válidos para todos os pontos (0...k), o BEST ajusta os dados para um mínimo de cinco pontos a um máximo de $\mathrm{N}_{\text {tot }}$. Para cada subconjunto de dados que contém os $\mathrm{k}$ primeiros pontos, o BEST estima a sorvidade $\mathrm{S}(\mathrm{k})$, a condutividade hidráulica $K_{s}(k)$ em função de $S(k)$ e da Eq. 13 para um tempo máximo $\mathrm{t}_{\max }(\mathrm{k})$ definido como:

$$
\mathrm{t}_{\max }=\frac{1}{4(1-b)^{2}} \cdot \mathrm{t}_{\mathrm{grav}}
$$

sendo $t_{\text {grav }}$ o tempo gravimétrico definido por Philip (1969). $\mathrm{O} \mathrm{t}_{\max }(\mathrm{k})$ é o tempo máximo para o qual as expressões para o regime transitório são consideradas válidas; logo, o tempo mais longo do subconjunto de dados $t_{k}$ é comparado com $t_{\max }(k)$. Os valores de $\mathrm{S}(\mathrm{k})$ e $\mathrm{K}_{\mathrm{s}}(\mathrm{k})$ são considerados válidos para $\mathrm{t}_{\mathrm{k}}$ menores que $t_{\max }(k)$. Dos valores que cumprirem esta condição, serão escolhidos aqueles correspondentes ao maior k.

Após a determinação de $\theta_{\mathrm{s}}$ e $\mathrm{K}_{\mathrm{s}}$, o parâmetro $\mathrm{h}_{\mathrm{g}}$ é obtido pela seguinte equação (Lassabatère et al., 2006):

$$
\mathrm{h}_{\mathrm{g}}=\frac{\mathrm{S}^{2}}{\mathrm{c}_{\mathrm{p}}\left(\theta_{\mathrm{s}}-\theta_{0}\right)\left[1-\left(\frac{\theta}{\theta_{\mathrm{s}}}\right)^{\mathrm{n}}\right] \cdot \mathrm{K}_{\mathrm{s}}}
$$

na qual $c_{p}$ é um parâmetro que depende apenas dos parâmetros de formas $n, m$ e $\eta$ dos modelos (de Condappa et al., 2002; Lassabatère et al., 2006):

$$
\mathrm{c}_{\mathrm{p}}=\Gamma\left(1+\frac{1}{\mathrm{n}}\right) \cdot\left[\frac{\Gamma\left(\mathrm{n} \eta+\frac{1}{\mathrm{n}}\right)}{\Gamma(\mathrm{n} \eta)}+\frac{\Gamma\left(\mathrm{n} \eta+\mathrm{m}-\frac{1}{\mathrm{n}}\right)}{\Gamma(\mathrm{n} \eta+\mathrm{m})}\right]
$$

sendo $\Gamma$ a função gama clássica.

\section{Escalas características}

Durante o processo de infiltração tridimensional os fatores que podem afetar o fluxo de entrada de água no solo, são: a geometria da fonte de água, a capilaridade e a gravidade; referidos fatores são caracterizados pelas escalas de comprimento capilar, $\lambda_{c}[\mathrm{~L}]$ (White $\&$ Sully, 1987) e o raio característico de poros hidraulicamente ativos, $\lambda_{\mathrm{m}}[\mathrm{L}]$ (Philip, 1987) determinados, respectivamente, pelas equações:

$$
\begin{aligned}
& \lambda_{\mathrm{c}}=\frac{\delta \mathrm{S}^{2}}{\left(\theta_{\mathrm{s}}-\theta_{0}\right) \mathrm{K}_{\mathrm{s}}} \\
& \lambda_{\mathrm{m}}=\frac{\sigma}{\rho_{\mathrm{a}} \mathrm{g} \lambda_{\mathrm{c}}}
\end{aligned}
$$

sendo $\sigma$ a tensão superficial da água $\left(0,0719 \mathrm{~N} \mathrm{~m}^{-1}\right), \rho_{\mathrm{a}}$ a massa específica da água $\left(10^{3} \mathrm{Kg} \mathrm{m}^{-3}\right)$, g a aceleração da gravidade $\left(9,81 \mathrm{~m} \mathrm{~s}^{-2}\right)$ e $\delta$ um parâmetro de forma da difusividade $(1 / 2 \leq \delta \leq \pi / 4)$, que foi considerado igual a 0,55 (White $\&$ Sully, 1987).

A escala de comprimento capilar (Eq. 25) representa a importância relativa das forças capilares em relação à gravidade, quando a água é transmitida de uma fonte através do solo, com umidade inicial $\theta_{0}$. O raio característico de poros (Eq. 26) define a dimensão média dos poros que participam do processo de infiltração submetida à pressão aplicada h; quanto maior for o raio característico, $\lambda_{\mathrm{m}}$, maior é o efeito da gravidade em comparação ao da capilaridade.

\section{RESULTADOS E DISCUSSÃO}

As curvas granulométricas para cada classe textural são apresentadas na Figura 2. As massas específicas das amostras de solo foram: $1,22,1,16,1,12 \mathrm{~g} \mathrm{~cm}^{-3}$, para as respectivas

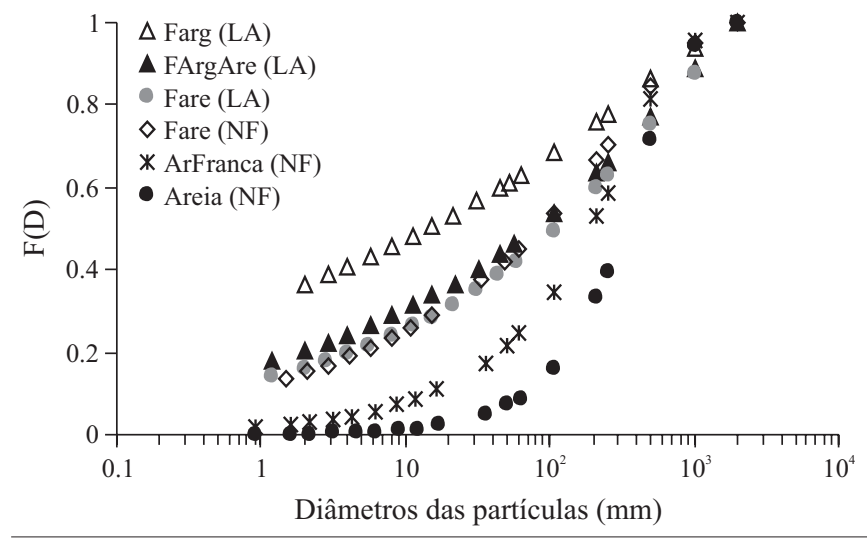

Figura 2. Curvas granulométricas das seis amostras de solo 
classes texturais do Latossolo Amarelo, FArg (LA), FArgAre (LA) e FAre (LA); e 1,30, 1,36 e 1,56 $\mathrm{g} \mathrm{cm}^{-3}$ para as respectivas classes texturais do Neossolo Flúvico, FAre (NF), ArFranca (NF) e Areia (NF).

\section{Parâmetros de forma $M, N, m, n$ e $\boldsymbol{\eta}$}

Os parâmetros de forma ( $\mathrm{M}, \mathrm{N}$ e seu produto $\mathrm{MN}$ ) das curvas de distribuição das partículas (Eq. 3) são maiores para os solos de frações mais arenosas, enquanto os parâmetros de escala do diâmetro das partículas $\mathrm{D}_{\mathrm{g}}$ foram menores (Tabela 1). FAre (LA) e FAre (NF) apresentam textura semelhante e, assim, valores semelhantes de $\mathrm{M}$ e $\mathrm{N}$; eles só diferem significamente no parâmetro de escala do diâmetro das partículas $\mathrm{D}_{\mathrm{g}}$; esta diferença pode ser atribuída à distribuição dos tamanhos das partículas da fração areia, nos dois solos. De acordo com a Figura 2, o FAre (NF) apresentou uma proporção maior de areia fina $(100$ - $250 \mathrm{~mm})$ que o FAre (LA). Para diâmetros menores que $50 \mathrm{~mm}$ as duas curvas foram idênticas.

Tabela 1. Parâmetros de forma das curvas de distribuição das partículas

\begin{tabular}{lcccr}
\hline Classes de solos & $\mathbf{M}$ & $\mathbf{N}$ & $\mathbf{M . N}$ & $\mathbf{D}_{\mathbf{g}}(\boldsymbol{\mu} \mathbf{m})$ \\
FArg (LA) & 0,07 & 2,16 & 0,16 & 1159,9 \\
FArgAre (LA) & 0,11 & 2,25 & 0,25 & 1324,1 \\
FAre (LA) & 0,12 & 2,28 & 0,28 & 1260,6 \\
FAre (NF) & 0,14 & 2,32 & 0,32 & 715,9 \\
ArFranca (NF) & 0,24 & 2,64 & 0,64 & 556,7 \\
Areia (NF) & 0,35 & 3,09 & 1,09 & 576,7 \\
\hline
\end{tabular}

Lassabatère et al. (2006) utilizaram o mesmo procedimento (Eq. 3) para obterem $\mathrm{N}$ e $\mathrm{D}_{\mathrm{g}}$ em três solos diferentes: um solo siltoso extremamente fino (Roujan); um solo estruturado com partículas maiores que $2 \mathrm{~mm}$ (Django Reinhardt) e um solo com aspectos texturais e estruturais intermediários aos dois primeiros solos (Chernobyl). Os valores de $\mathrm{N}$ encontrados foram iguais a 2,42, 3,92 e 3,48 para Roujan, Chernobyl e Django Reinhardt, respectivamente. Para $\mathrm{D}_{\mathrm{g}}$, esses autores encontraram valores iguais 171, 177 e $779 \mathrm{~mm}$ para os respectivos solos. de Condappa et al. (2002), analisando situações texturais extremas, encontraram valores de $\mathrm{N}$ iguais a 2,79 (para um solo arenoso de Grenoble) e 2,22 (para um solo argiloso - Yolo Light Clay).

Os valores de $\mathrm{N}$ encontrados para os dois solos (Tabela 1) estão em concordância com os valores estudados tanto por Lassabatère et al. (2006), como por de Condappa et al. (2002).

Os parâmetros m e n, tal como esperado e por se tratar de parâmetros que dependem da textura, apresentaram o mesmo comportamento que os parâmetros $\mathrm{M}$ e $\mathrm{N}$ de $\mathrm{F}(\mathrm{D})$, ou seja, foram maiores para as classes texturais com maior teor de areia, enquanto os valores de $\eta$ para $K(\theta)$ e de $c_{p}$ foram menores (Tabela 2). Esta relação inversa entre n e $\eta$ se deve ao próprio formalismo matemático para se obter $\eta$ (Eq. 9).

Os valores de n encontrados por Lassabatère et al. (2006) foram iguais a 2,20, 2,97 e 2,65 para Roujan, Chernobyl e Django Reinhardt, respectivamente, enquanto para $\eta$, eles encontraram valores iguais 13,2, 5,06 e 6,07, para os respectivos solos.
Tabela 2. Resultado dos parâmetros de forma das funcionais de $h(\theta)$ e $K(\theta)$

\begin{tabular}{lcccc}
\hline Classes de solos & $\mathbf{m}$ & $\mathbf{n}$ & $\boldsymbol{\eta}$ & $\mathbf{c}_{\mathbf{p}}$ \\
FArg (LA) & 0,04 & 2,08 & 28,76 & 2,72 \\
FArgAre (LA) & 0,06 & 2,12 & 20,23 & 2,57 \\
FAre (LA) & 0,06 & 2,13 & 18,16 & 2,52 \\
FAre (NF) & 0,07 & 2,16 & 15,67 & 2,44 \\
ArFranca (NF) & 0,13 & 2,31 & 9,54 & 2,13 \\
Areia (NF) & 0,21 & 2,54 & 6,70 & 1,88 \\
\hline
\end{tabular}

\section{Parâmetros de normalização $\boldsymbol{\theta}_{\mathrm{s}}, \mathbf{K}_{\mathrm{s}} \mathbf{e} \mathbf{h}_{\mathrm{g}}$}

Os testes de infiltração (Figura 3 ) estão agrupados de acordo com a textura do solo. As comparações das lâminas de água medidas nas diferentes classes texturais, demonstram diferenças qualitativas dos efeitos texturais e estruturais no fluxo de água.

A duração das infiltrações no Latossolo Amarelo foi em torno de $900 \mathrm{~s}$ para $45 \mathrm{~mm}$ do total da lâmina acumulada, no FArg (LA), de 500 s para 90 mm, no FArgAre (LA), e de $270 \mathrm{~s}$ para $85 \mathrm{~mm}$, no FAre (LA); por outro lado, no Neossolo Flúvico a duração foi em torno de $1700 \mathrm{~s}$ para $40 \mathrm{~mm}$, no FAre (NF), de $1000 \mathrm{~s}$ para $60 \mathrm{~mm}$, na ArFranca (NF), e de $200 \mathrm{~s}$ para $90 \mathrm{~mm}$, na Areia (NF) (Figura 3); desta forma, é possível observar uma variabilidade mais significativa dos fluxos de infiltração no Neossolo Flúvico.

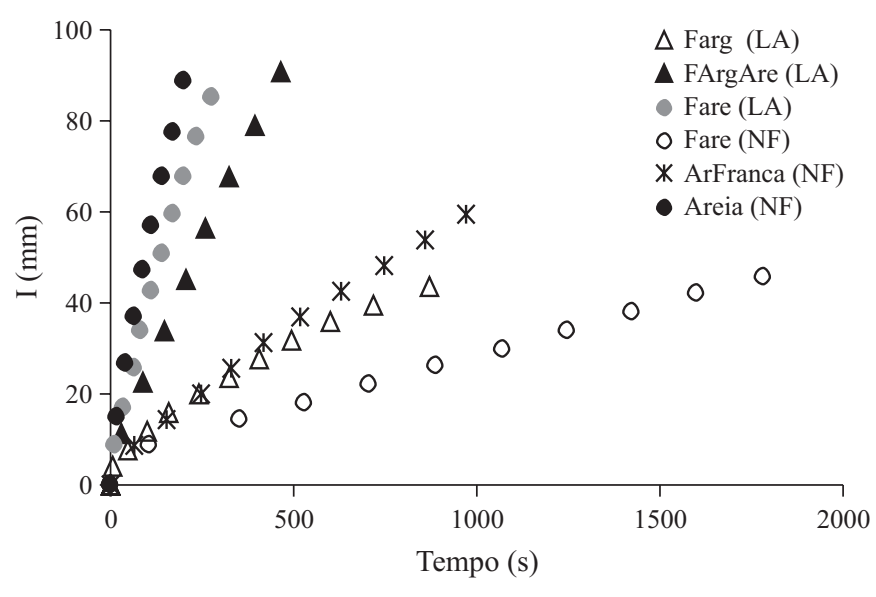

Figura 3. Curvas de infiltração acumulada I (mm) em função do tempo para os dois solos

Apresentam-se, na Tabela 3, os valores de $\mathrm{S}, \mathrm{K}_{\mathrm{s}}$, EQM, $\theta_{0}, \theta_{\mathrm{s}}, \mathrm{h}_{\mathrm{g}}$ e $\lambda_{\mathrm{m}}$, para os dois solos. As análises dessas propriedades são bem distintas entre as diferentes classes texturais. Comparando-se as três classes texturais amostradas em cada solo, observa-se que os valores de $\mathrm{S}$ e $\mathrm{K}_{\mathrm{s}}$ aumentam com o teor de areia, identificando o FAre (LA) como a mais permeável para o Latossolo Amarelo e a Areia (NF) para o Neossolo Flúvico.

Verifica-se, ainda na Tabela 3, que no Latossolo Amarelo o $\lambda_{\mathrm{m}}$ (raio característico de poros hidraulicamente funcionais) (Eq. 25) aumenta com o teor de areia, enquanto para o Neossolo Flúvico diminui. No Latossolo Amarelo o aumento nos raios dos poros que efetivamente transportam água, $\lambda_{\mathrm{m}}$, explica o aumento de $\mathrm{K}_{\mathrm{s}}$ e de $\mathrm{S}$ com a diminuição das massas específicas $\left\{1,22,1,16,1,12 \mathrm{~g} \mathrm{~cm}^{-3}\right.$ para FArg (LA), FArgAre 
Tabela 3. Valores de $S, K_{s^{\prime}}$ EQM, $\theta_{0^{\prime}} \theta_{s^{\prime}} h_{g}$ e $\lambda_{m^{\prime}}$ para os dois solos

\begin{tabular}{|c|c|c|c|c|c|c|c|}
\hline Classes de solos & $S\left(\mathrm{~mm} \mathrm{~s}^{-1 / 2}\right)$ & $\mathrm{K}_{\mathrm{s}}\left(\mathrm{mm} \mathrm{s}^{-1}\right)$ & EQM & $\theta_{0}\left(\mathrm{~cm}^{3} \mathrm{~cm}^{-3}\right)$ & $\theta_{\mathrm{s}}\left(\mathrm{cm}^{3} \mathrm{~cm}^{-3}\right)$ & $h_{g}(\mathrm{~mm})$ & $\overline{\lambda_{m}(\mathrm{~mm})}$ \\
\hline FArg (LA) & 0,79 & 0,01 & 1,92 & 0,10 & 0,37 & $-97,9$ & 0,05 \\
\hline FArgAre (LA) & 1,79 & 0,05 & 3,54 & 0,05 & 0,44 & $-62,1$ & 0,08 \\
\hline Fare (LA) & 2,07 & 0,13 & 3,56 & 0,07 & 0,40 & $-39,2$ & 0,14 \\
\hline Fare (NF) & 0,49 & 0,02 & 8,33 & 0,05 & 0,47 & $-14,5$ & 0,38 \\
\hline ArFranca (NF) & 0,79 & 0,03 & 2,88 & 0,08 & 0,36 & $-36,6$ & 0,17 \\
\hline Areia (NF) & 2,48 & 0,19 & 0,72 & 0,03 & 0,37 & $-51,9$ & 0,14 \\
\hline
\end{tabular}

(LA) e FAre (LA)\}. No Neossolo Flúvico a redução do $\lambda_{\mathrm{m}}$ é compensada pelo aumento na densidade de poros (quantidade por unidade de área); assim, embora o tamanho do $\lambda_{\mathrm{m}}$ tenha diminuído com o aumento da massa especifica do solo, a quantidade de poros hidraulicamente ativos aumentou; isto mostra a importância da estrutura do solo, visto que solos com a mesma textura, como o FAre (LA) e o FAre (NF) apresentaram comportamentos bem distintos. Os valores de $\mathrm{S}$ e $\mathrm{K}_{\mathrm{s}}$ no FAre (LA) são bem superiores aos do FAre (NF) (Tabela 3); tal diferença pode ser atribuída tanto à distribuição dos tamanhos das partículas da fração de areia fina como à massa específica nos dois solos $\left(1,12 \mathrm{~g} \mathrm{~cm}^{-3}\right.$ para o FAre (LA) e $1,30 \mathrm{~g} \mathrm{~cm}^{-3}$ para o FAre (NF)); esta combinação proporciona poros com classes e quantidades diferentes; novamente isto pode ser inferido por meio do valor do $\lambda_{\mathrm{m}}$. Para o FAre (LA) o $\lambda_{\mathrm{m}}$ é aproximadamente três vezes menor que o do FAre (NF), mostrando que o FAre (LA) tem maior quantidade de poros hidraulicamente funcionais que o FAre (NF).

Os valores do raio característico de poros hidraulicamente funcionais $\left(\lambda_{\mathrm{m}}\right)$ encontrados variaram de 0,05 a $0,38 \mathrm{~mm}$ (Tabela 3) e estão de acordo com os valores reportados em outros trabalhos (White \& Sully, 1987; Sauer et al., 1990; Thony et al., 1991; Cook \& Boeren, 1994; Angulo-Jaramillo et al., 1997; Borges et al., 1999).

\section{Curvas de retenção e da condutividade hidráulica da água no solo}

Definidos os parâmetros de forma (m ou n e $\eta$ ) e de normalização $\left(\theta_{\mathrm{s}}, \mathrm{K}_{\mathrm{s}}\right.$ e $\left.\mathrm{h}_{\mathrm{g}}\right)$ para as Eqs. 1 e 2, construíram-se as curvas $\theta(\mathrm{h})$ (Figura 4A) e K( $\theta$ ) (Figura 4B) para as seis classes texturais; em cada curva, o ponto de origem, $\theta_{\mathrm{s}}$, foi determinado durante os ensaios de infiltração; elas apresentam claramente a inflexão característica do modelo, logo após a saturação, correspondente ao valor do potencial de entrada de ar $\left(h_{g}\right)$ na matriz porosa do solo.

Analisando-se as situações extremas, FArg (LA) e Areia (NF), observa-se que a variação nos teores de umidade do FArg (LA) são bem menores que na Areia (NF), porém a sua capacidade de retenção é bem maior. Para $h=1 \mathrm{~m}$, as umidades do solo correspondentes a FArg (LA) e a Areia (NF) foram iguais a 0,32 e $0,08 \mathrm{~cm}^{3} \mathrm{~cm}^{-3}$, respectivamente, enquanto para $\mathrm{h}=10 \mathrm{~m}$, os valores de $\theta$ foram iguais a $0,26 \mathrm{e}$ $0,02 \mathrm{~cm}^{3} \mathrm{~cm}^{-3}$, respectivamente. A maior proximidade das partículas do FArg (LA), faz com que os efeitos de adsorção e capilaridade sejam mais intensos que na Areia (NF), retendo maior quantidade de água.

A ArFranca (NF) e Areia (NF), via de regra, apresentam menor capacidade de retenção de água sempre que se apro- ximam da saturação $\left(\theta=\theta_{\mathrm{s}}\right)$; além da baixa capacidade de retenção, a Areia (NF) possui boa parte do espaço poroso formado por uma grande quantidade de poros hidraulicamente funcionais, fato este relacionado à distribuição dos tamanhos das partículas dessas classes texturais, que apresentam forma unimodal com pequeno desvio (Figura 2).
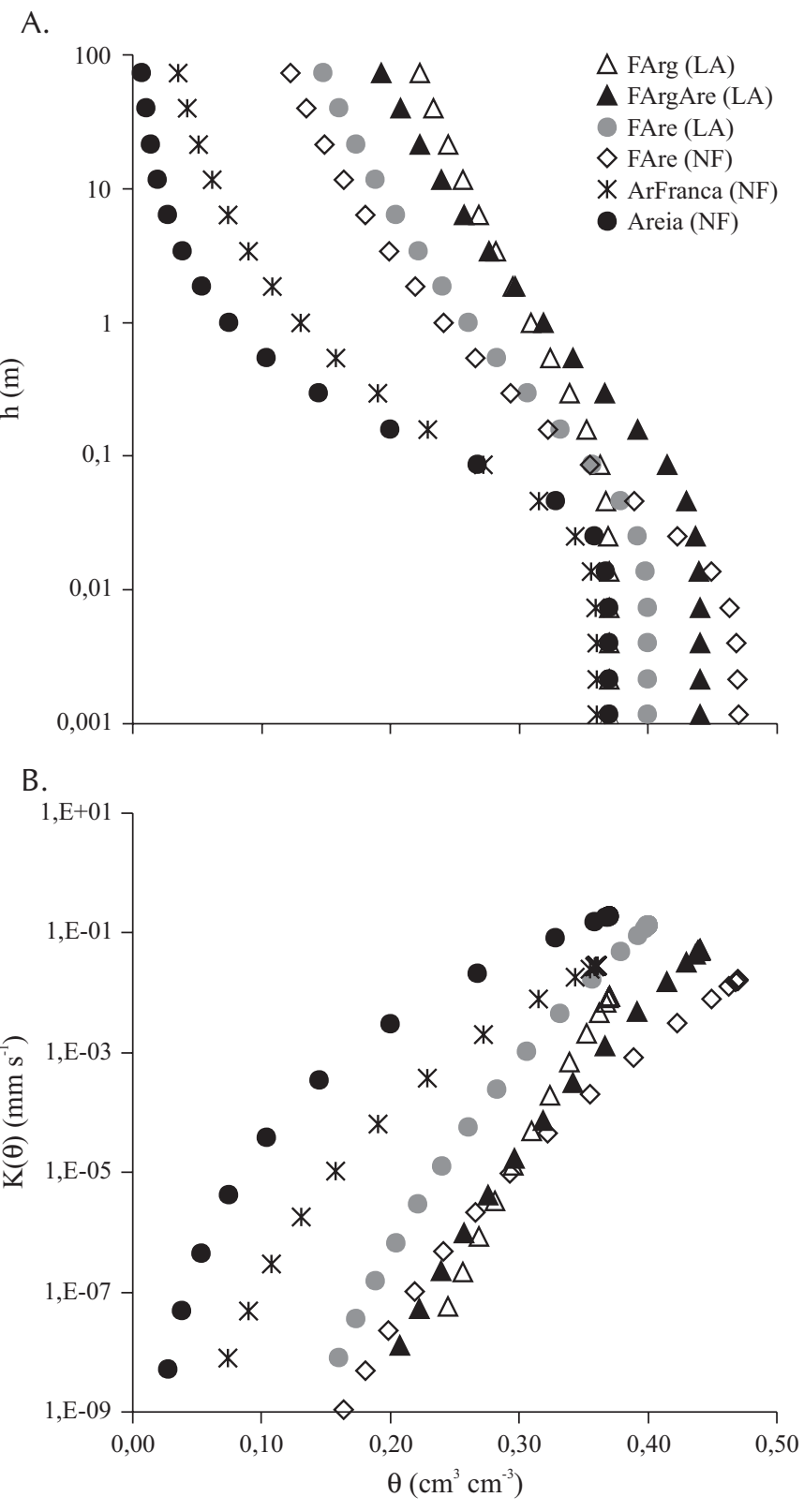

Figura 4. Curvas de retenção (A) e da condutividade hidráulica (B) para os dois solos 
A análise das curvas de condutividade hidráulica ajuda a entender melhor o comportamento hidráulico nos dois solos (Figura 4B). Para FArg (LA), FArgAre (LA) e FAre (NF), por exemplo, observa-se inversão de comportamento nas curvas de $\mathrm{K}(\theta)$ a partir do conteúdo volumétrico de água igual a $0,30 \mathrm{~cm}^{3} \mathrm{~cm}^{-3}$. Para $\theta<0,30 \mathrm{~cm}^{3} \mathrm{~cm}^{-3}$, os valores de $\mathrm{K}(\theta)$ para FArg (LA) são menores que os do FArgAre (LA) e do FAre (NF), enquanto para $\theta>0,30 \mathrm{~cm}^{3} \mathrm{~cm}^{-3}$ esta condição se inverte e os valores de $K(\theta)$ de FArg (LA) passam a ser maiores.

\section{CONCLUSÕES}

1. O método Beerkan forneceu valores aceitáveis para a sorvidade (S) e para a condutividade hidráulica à saturação $\left(\mathrm{K}_{\mathrm{s}}\right)$ para os dois solos, além de fornecer ajustes precisos das infiltrações acumuladas.

2. O método Beerkan se manteve apropriado, robusto e inteiramente adaptado para modelar a infiltração tridimensional no campo, além de permitir a caracterização hidrodinâmica de diferentes solos e em vários pontos, por ser de fácil utilização, rápido e de baixo custo, tornando mais fácil o estudo da variabilidade espacial das propriedades hidráulicas dos solos.

\section{AGRADECIMENTOS}

Agradecimentos a CAPES (Projeto de Cooperação internacional CAPES-COFECUB, No 350/01); à Fundação de Apoio a Pesquisa do Estado da Paraíba - FAPESQ (processo No 003/05, Edital 002/03 - FAPESQ/MCT/CNPq, convênio 002/2003) e ao CNPq (Processos Nos 350277/2005-2, 309986/2006-0, 373089/2006-6 e 373139/2006-3) pela concessão de bolsas e dos recursos necessários ao desenvolvimento deste trabalho.

\section{LITERATURA CITADA}

Angullo-Jaramillo, R.; Moreno, F. B. E.; Clothier, J. L.; Thony, G.; Vachaud, E. Fernadez-Boy, E. and Cayuela, J. A. Seasonal variation of hydraulic properties of soils measured using a tension disk infiltrometer. Soil Science Society of American Journal, v.61, p.27-32, 1997.

ABNT - Associação Brasileira de Normas Técnicas. ABNT NBR 7181. Análise granulométrica. Rio de Janeiro: ABNT, 1984. 13p.

Borges, E.; Antonino, A. C. D.; Dall’olio, A.; Audry, P. E.; Carneiro, C. J. G. Determinação da condutividade hidráulica e da sorvidade de um solo não-saturado utilizando-se permeâmetro a disco. Pesquisa Agropecuária Brasileira, v.34, n.11. p.2083-2089, 1999.

Braud, I.; de Condappa, D.; Soria Uglade, J. M.; Haverkamp, R.; Angulo-Jaramillo, R.; Galle, S.; and Vauclin, M. Use of scaled forms of the infiltration equation for the estimation of unsaturated soil hydraulic properties (the Beerkan method). European Journal of Soil Science, v.56, p.361-374, 2005.
Brooks, R. H.; Corey, A. T. Hydraulic properties of porous media. Hydrology Paper, n.3, Fort Collins: Colorado State University, 1964, 27p.

Burdine, N. T. Relative permeability calculations from pore-size distribution data. American Institute Mining and Metallurgy Engineering, v.198, p.71-77, 1953.

Cook, F. J.; Broeren, A. Six methods for determining sorptivity and hydraulic conductivity with disc permeameters. Soil Science, v.157, p.3-11, 1994.

de Condappa, D.; Soria Ugalde, J. M.; Angulo-Jaramillo, R.; Haverkamp, R. 2002. Méthode Beerkan. Caractérisation des propriétés hydrodynamiques des sols non saturés. Rapport interne Hydrologie de la Zone Non Saturés - LTHE, Grenoble: Université de Grenoble, 2002, 82p.

EMBRAPA - Empresa Brasileira de Pesquisa Agropecuária. Centro Nacional de Pesquisa de Solos. Sistema brasileiro de classificação de solos. Brasília: Embrapa - Serviço de Produção da Informação; Rio de Janeiro, Embrapa Solos, 1999, 412p.

Fuentes, C. Approche fractale des transferts hydriques dans les sols non-saturés. Grenoble: Université Joseph Fourier, 1992. 444p. Tese Doutorado

Fuentes, C.; Vauclin, M.; Parlange, J. Y.; Haverkamp, R. Soil water conductivity of a fractal soil. In: Baveye, P.; Parlange, J. Y.; Stewart, B. A. (eds.). Fractals in soil science. Boca Raton: CRC, 1998. cap. 11, p.333-340.

Haverkamp, R.; Parlange, J. Y. Predicting the water retention curve from particle size distribution: I Sandy soils without organic matter. Soil Science, v.142, p.325-335, 1986.

Haverkamp, R.; Parlange, J. Y.; Cuenca, R.; Ross, P. J.; Steenhuis, T. S. Scaling of the Richards equation and its application to watershed modeling. In: Sposito, G. (ed.). Scale dependence and scale invariance in hydrology. Cambridge: Cambridge University Press, 1998. p.190-223.

Haverkamp, R.; Ross, P. J.; Smettem, K. R. J.; Parlange, J. Y. Three dimensional analysis of infiltration from the disc infiltrometer. 2. Physically based infiltration equation. Water Resources Research, v.30, p.2931-2935, 1994.

Hillel, D. Environmental soil physics. New York: Academic Press, 1998. 771p.

Lassabatère, L.; Angulo-Jaramillo, R.; Soria, J. M.; Cuenca, R.; Braud, I.; Haverkamp, R. Beerkan estimation of soil transfer parameters through infiltration experiments BEST. Soil Science Society of American Journal, v.70, p.521-532, 2006.

Marquardt, D. W. An algorithm for least squares estimation of non linear parameters. SIAM Journal on Applied Mathematics, v.11, p.431-441, 1963.

Minasny, B.; MacBratney, A. B. The efficiency of various approaches to obtaining estimates of soil hydraulic properties. Geoderma, v.107, p.55-70, 2002.

Minasny, B.; MacBratney, A. B.; Bristow, K. L. Comparison of different approaches to the development of pedotransfer functions for water-retention curves, Geoderma, v.93, p.225-253, 1999.

Philip, J. R. Theory of infiltration. In: Chow, V. T. (ed.). Advances in Hydroscience. New York: Academic Press, 1969. vol.5, p.215-296. 
Philip, J. R. The quasi-linear analysis, the scattering analog, and other aspects of infiltration and seepage. In: Y. S. Fok (ed.), Infiltration development and application, Honolulu: Water Resources Research Center, 1987, p.1-27.

Sauer, T. J.; Clothier, B. E.; Daniel, T. C. Surface measurements of the hydraulic properties of tilled and untilled soil. Soil \& Tillage Research, v.15, p.359-369, 1990.

Thony, J. L.; Vachaud, G.; Clothier, B. E.; Angulo-Jaramillo, R. Field measurement of the hydraulic properties of soil. Soil Technology, v.4, p.111-123, 1991.

van Genuchten, M. Th. A closed-form equation for predicting the hydraulic conductivity of unsaturated soils. Soil Science Society of American Journal, v.44, p.892-898, 1980.
Wagner, B.; Tarnawski, V. R.; Hennings, V.; Muller, U.; Wessolek, G.; Plagge, R. Evaluation of pedo-transfer functions for unsaturated soil hydraulic conductivity using an independent data set. Geoderma, v.102, p.275-297, 2001.

White, I.; Sully, M. J. Macroscopic and microscopic capillary length and times scales from field infiltration. Water Resources Research, v.23, n.8, p.1514-1522, 1987.

Zataráin, F.; Fuentes, C.; Haverkamp, R.; Antonio, A. C. D. Predicción de la forma de la característica de humedad del suelo a partir de la curva granulométrica. In: Congreso Nacional de Irrigación, 13, 2003, Zacatecas. Resumos ... Zacatecas: ANEI, 2003. Desarrollo y transferencia de tecnología de riego y drenaje, v.2, p.212-219. 